\title{
Novel Preamble-Based Channel Estimation for OFDM/OQAM Systems
}

\author{
Jinfeng Du† \\ ACCESS Linnaeus center \\ School of Electrical Engineering \\ KTH - Royal Institute of Technology, Stockholm, Sweden \\ Email: jinfeng@kth.se
}

\author{
Svante Signell, Senior Member, IEEE \\ Department of Communication Systems \\ School of Information and Communication Technology \\ KTH - Royal Institute of Technology, Stockholm, Sweden \\ Email: srs@kth.se
}

\begin{abstract}
OFDM/OQAM has been considered as an attractive alternative to classic OFDM with cyclic prefix (CP) over doubly dispersive channels. By utilising well designed pulse shapes and removing CP, OFDM/OQAM has the advantage of reduced outof-band energy and a theoretically higher spectral efficiency. However, channel estimation over doubly dispersive channels has been a big problem for OFDM/OQAM due to the nonorthogonality between the real and imaginary parts of its modulated signals. Therefore conventional channel estimation (CE) methods used for OFDM cannot be directly applied to OFDM/OQAM. Recently a preamble-based CE method - interference approximation method (IAM) - has been proposed to ease this task. By treating the intrinsic interference from neighbour symbols as known information, two heuristic preamble sequences have been constructed based on tentative observations, which turn out to be suboptimal. In this paper, we present a general theoretical framework for IAM preamble design and apply it to identify the optimal IAM preamble sequence which results in a higher gain. Numerical results have verified the effectiveness of the theoretical framework and a gain of $2.4 \mathrm{~dB}$ against $\mathrm{CP}$ OFDM has been demonstrated with the new preamble in various doubly dispersive channels with a QPSK modulation.
\end{abstract}

\section{INTRODUCTION}

Multicarrier communication technologies are promising candidates to realise high data rate transmission in Beyond 3G and furture wireless systems where the channel is normally doubly dispersive. Contrary to the classic OFDM system which uses a cyclic prefix (CP) to combat time dispersion, OFDM/OQAM [1]-[3] utilises well designed pulse shapes and/or system lattice but no $\mathrm{CP}$, and hence has the advantage of reduced out-of-band energy and a theoretically higher spectral efficiency. Performance evaluation of OFDM/OQAM has already illustrated promising advantage [4], [5] and it has already been introduced in the TIA's Digital Radio Technical Standards [6] and been considered in WRAN (IEEE 802.22) [5], but in the latter case it was recently pulled out to appendices pending further study partially due to its difficulties in channel estimation (CE).

Due to the non-orthogonality between the real and imaginary parts in OFDM/OQAM signals, the demodulated realvalued OFDM/OQAM symbol always has imaginary-valued

This work was partially funded by VINNOVA and Wireless@KTH.

$\dagger$ This work was submitted when Jinfeng Du was with Department of Communication System, School of ICT, KTH. intrinsic interference from neighbouring symbols [3]. This hinders conventional CE methods for OFDM from beeing directly applied to OFDM/OQAM. Therefore CE has been a big problem for OFDM/OQAM in dispersive channels and attracted numerous research efforts. In order to reduce the intrinsic interference to a minimum, a pilot-based CE scheme has been proposed in [7] and a preamble-based CE method in [8], where in both cases a group of adjacent symbols are carefully selected so that the intrinsic interference at the central symbol position can be greatly reduced. On the contrary, by treating the intrinsic interference as known information and consequently forming a complex-valued "pseudo pilot", a preamble-based CE scheme - interference approximation method (IAM) - has been proposed recently in [9]-[11] and verified through purely time dispersive channels. An ideal yet unrealistic CE method proposed in [9] produces a performance upper bound. A real-valued preamble sequence, denoted IAM-R in the following, has been proposed in [10] which increases the power of the pseudo pilot by making the imaginary-valued interference terms constructively added ${ }^{1}$. In [11] the real-valued preamble symbol has been replaced by an imaginary one, hence named IAM-I in the following, so that the transmitted symbol and its associated intrinsic interference are both imaginary-valued and constructively added. The two heuristic preamble sequences (IAM-R and IAM-I), however, are suboptimal since they were constructed based on tentative observations. Additionally their performance over frequency dispersive channels, which is common in mobile communication, has not been demonstrated.

Motivated by the principle of IAM method and awareness of the suboptimal nature of the two IAM methods in [10], [11], we have in this paper formulated a general theoretical framework for IAM preamble design. As a consequence the optimal IAM preamble sequence has been identified which results in a higher performance gain. The effectiveness of the theoretical framework and the superiority of proposed preamble has thereafter been verified by numerical simulation with time and frequency dispersive channels.

The rest of this paper is organised as follows. Section II

\footnotetext{
${ }^{1}$ Here the term 'constructively add' means that all the elements within the summation have the same sign.
} 
presents the system model and the general theoretical framework for IAM preamble design. In Section III we revisit the two heuristic preamble sequences under the framework and then derive an optimal IAM preamble sequence. Simulation results under various doubly dispersive channels are shown in Section IV and conclusions are drawn in Section V.

\section{SYSTEM MODEL}

The transmitted signal in CP-OFDM and OFDM/OQAM systems can be written in the following analytic form

$$
s(t)=\sum_{n=-\infty}^{+\infty} \sum_{m=0}^{N-1} a_{m, n} g_{m, n}(t)
$$

where $a_{m, n}(m=0,1, \ldots, N-1, n \in \mathbb{Z})$ denotes the symbol conveyed by the sub-carrier of index $m$ during the symbol time of index $n$, and $g_{m, n}(t)$ represents the synthesis basis which is obtained by time-frequency translation of the prototype function $g(t)$. In CP-OFDM systems

$$
g_{m, n}(t)=\left\{\begin{array}{cc}
e^{j 2 \pi m F t}, & n T_{o}-T_{c p} \leq t \leq n T_{o}+T \\
0, & \text { otherwise }
\end{array}\right.
$$

where $F=1 / T$ is inter-carrier frequency separation, $T_{c p}$ is the length of $\mathrm{CP}, T_{o}=T+T_{c p}$ is CP-OFDM symbol duration and $a_{m, n}$ are complex valued symbols. In OFDM/OQAM systems

$$
g_{m, n}(t)=e^{\phi_{0}+j(m+n) \pi / 2} e^{j 2 \pi m \nu_{0} t} g\left(t-n \tau_{0}\right), \quad \nu_{0} \tau_{0}=1 / 2
$$

where $\phi_{0}$ is an additional phase shift and $g(t)$ is the well designed pulse shape prototype. Here the transmitted symbols $a_{m, n}$ are real-valued with symbol duration $\tau_{0}$ and intercarrier spacing $\nu_{0}$ respectively. Two kinds of realisations of OFDM/OQAM are of practical interest. One can either set $\nu_{0}=F, \tau_{0}=T / 2$ as in [12], [13] or set $\tau_{0}=T, \nu_{0}=F / 2$ as in [14]. We use the former approach.

After passing through the doubly dispersive channel, the received signal (noise is omitted here for simplicity) can be written as

$$
\begin{aligned}
r(t) & =\int h(\tau, t) s(t-\tau) d \tau=\iint H(\tau, \nu) s(t-\tau) e^{j 2 \pi \nu t} d \nu d \tau \\
& =\sum_{m, n} a_{m, n} \iint H(\tau, \nu) g_{m, n}(t-\tau) e^{j 2 \pi \nu t} d \nu d \tau
\end{aligned}
$$

where $h(\tau, t)$ is the channel impulse response and $H(\tau, \nu)$ is its Fourier transform with respect to $t$. The integration interval depends on the maximum time spread $\tau_{\max }$ and the maximum Doppler frequency $f_{D}$. Without loss of generality, we assume $a_{k, l}$ is the symbol to be detected, with the corresponding demodulation output

$$
\hat{a}_{k, l}=<r(t), g_{k, l}(t)>\triangleq \int r(t) g_{k, l}^{*}(t) d t
$$

Define the ambiguity function of $g(t)$ the same way ${ }^{2}$ as in [3]

$$
A_{g}(\tau, \nu)=\int_{\mathbb{R}} e^{-j 2 \pi \nu t} g(t+\tau / 2) g^{*}(t-\tau / 2) d t,
$$

\footnotetext{
${ }^{2}$ Another definition for the ambiguity function only differs by a phase shift.
}

then for OFDM/OQAM the correlation between transmit and receive pulse shapes can be written as in (3), shown on the top of next page. Note that the additional phase shift $\phi_{0}$ in $g_{m, n}(t)$ is totally cancelled out in this step. Apply (3) to (2) and do the variable substitution $p=m-k$ and $q=n-l$, we get

$$
\begin{gathered}
\hat{a}_{k, l}=\sum_{p, q} a_{k+p, l+q} j^{p+q+p(q+2 l)} \iint_{\nu_{0}} H(\tau, \nu) \\
\cdot A_{g}^{*}\left(q \tau_{0}+\tau, p \nu^{j \pi\left(q \tau_{0} \nu-p \nu_{0} \tau\right)} e^{j \pi\left(2 l \tau_{0} \nu-2 k \nu_{0} \tau+\tau \nu\right)} d \nu d \tau\right.
\end{gathered}
$$

Assume the ambiguity function $A_{g}(\tau, \nu)$ has relatively low variation around the lattice points $\left(q \tau_{0}, p \nu_{0}\right)$ over the integration interval ${ }^{3}$

$$
A_{g}^{*}\left(q \tau_{0}+\tau, p \nu_{0}+\nu\right) e^{j \pi\left(q \tau_{0} \nu-p \nu_{0} \tau\right)} \approx A_{g}^{*}\left(q \tau_{0}, p \nu_{0}\right)
$$

(4) can be rewritten as

$$
\begin{aligned}
& \hat{a}_{k, l}=\sum_{p, q} a_{k+p, l+q} j^{p+q+p(q+2 l)} A_{g}^{*}\left(q \tau_{0}, p \nu_{0}\right) \\
& \cdot \iint H(\tau, \nu) e^{j \pi\left(2 l \tau_{0} \nu-2 k \nu_{0} \tau+\tau \nu\right)} d \nu d \tau=a_{k, l}^{(c)} H_{k, l}^{(c)}
\end{aligned}
$$

where

$$
H_{k, l}^{(c)} \triangleq \iint H(\tau, \nu) e^{j \pi\left(2 l \tau_{0} \nu-2 k \nu_{0} \tau+\tau \nu\right)} d \nu d \tau
$$

represents the channel coefficient at $l$ th symbol and $k$ th subcarrier frequency, and

$$
a_{k, l}^{(c)} \triangleq \sum_{p, q} a_{k+p, l+q} j^{p+q+p(q+2 l)} A_{g}^{*}\left(q \tau_{0}, p \nu_{0}\right)
$$

indicates the superposition of the transmitted symbol $a_{k, l}$ and ISI/ICI components after demodulation at the lattice point $\left(l \tau_{0}, k \nu_{0}\right)$. Note that $a_{k, l}^{(c)}$ only depends on the pulse shape function $g(t)$ and the transmitted symbols $a_{p, q}$, and therefore can be calculated before transmission. Instead of using $a_{k, l}$ for $\mathrm{CE}$ by reducing as much as possible the power of the ISI/ICI terms as in [7], [8], the CE method IAM [10], [11] utilises the full knowledge of $a_{k, l}^{(c)}$ and treats the whole as a "pseudo pilot" to do estimation.

With noise taken into consideration, CE becomes

$$
\hat{H}_{k, l}^{(c)}=\frac{\hat{a}_{k, l}}{a_{k, l}^{(c)}}=H_{k, l}^{(c)}+\frac{w_{k, l}}{a_{k, l}^{(c)}}
$$

where $w_{k, l}$ is the noise term in the output of demodulation. Note that the larger the power of $a_{k, l}^{(c)}$ the better the estimation will be. Therefore, we focus on increasing the power of the ISI/ICI rather than reducing it.

\section{IAM PREAMBLE DESIGN REVISIT}

The frame structures of the IAM-R preamble [10] and the IAM-I preamble [11] are shown in Fig. 1, where the frame structure for CP-OFDM is also depicted. Note that the preamble length in OFDM/OQAM is $3 \tau_{0}$ instead of $2 \tau_{0}$ as in CP-OFDM. In an OFDM/OQAM system, the pulse shape $g(t)$ is chosen to have a very good time frequency localisation (TFL) property and therefore the ambiguity function $A_{g}(\tau, \nu)$

\footnotetext{
${ }^{3}$ This assumption holds when the channel is moderately dispersive $\left(\tau_{\max } \ll \tau_{0}\right.$ and $\left.f_{D} \ll \nu_{0}\right)$.
} 


$$
\begin{aligned}
& \int g_{m, n}(t-\tau) g_{k, l}^{*}(t) e^{j 2 \pi \nu t} d t=j^{m+n-k-l} e^{-j 2 \pi m \nu_{0} \tau} \int g\left(t-n \tau_{0}-\tau\right) g^{*}\left(t-l \tau_{0}\right) e^{j 2 \pi\left[(m-k) \nu_{0}+\nu\right] t} d t \\
& =j^{m+n-k-l} e^{-j 2 \pi m \nu_{0} \tau} e^{j \pi\left[(m-k) \nu_{0}+\nu\right]\left[(n+l) \tau_{0}+\tau\right]} A_{g}^{*}\left((n-l) \tau_{0}+\tau,(m-k) \nu_{0}+\nu\right)
\end{aligned}
$$

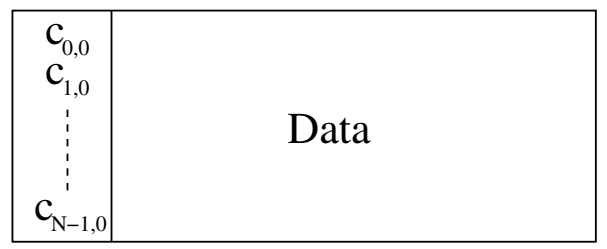

(a) Preamble structure used in CP-OFDM

\begin{tabular}{|rrr|l|}
\hline 0 & 1 & 0 & \\
0 & 1 & 0 & \\
1 & -1 & 1 & \\
1 & 1 & 1 & \\
1 & -1 & 1 & Data \\
1 & 1 & 1 & \\
1 & 1 & 1 & \\
0 & 1 & 0 & \\
\hline
\end{tabular}

\begin{tabular}{|c|c|}
\hline 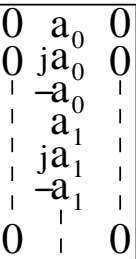 & Data \\
\hline
\end{tabular}

(b) IAM-R used in OFDM/OQAM

(c) IAM-I used in OFDM/OQAM

Figure 1. Frame structures for CP-OFDM and OFDM/OQAM: x-axis for time and y-axis for frequency. $\left|c_{k, 0}\right|=1$ and $a_{k} \in\{ \pm 1\}$.

attenuates fast both in time and frequency as $(\tau, \nu)$ deviates from the origin. Since only the surrounding symbols can introduce notable interference in $a_{k, l}^{(c)}$, the neighbouring two columns of 0 suppress the ISI to a very small amount [10]. With $a_{m, l-1}=a_{m, l+1}=0$ for $m=0 . . N-1$, (8) can be rewritten as

$$
a_{k, l}^{(c)}=\sum_{p} a_{k+p, l}(-1)^{p l} j^{p} A_{g}^{*}\left(0, p \nu_{0}\right)
$$

For a real and even pulse shape $g(t)$ with unit energy, the ambiguity function $A_{g}(\tau, \nu)$ has the properties:

$$
A_{g}( \pm \tau, \pm \nu)=A_{g}(\tau, \nu)=A_{g}^{*}(\tau, \nu), \quad A_{g}(0,0)=1
$$

This helps to simplify the following derivation.

\section{A. IAM-R}

For OFDM/OQAM the orthogonality within the real domain is ensured [3], i.e.,

$$
A_{g}\left(2 n \tau_{0}, 2 m \nu_{0}\right)=0 \text { for }(m, n) \neq(0,0)
$$

which indicates all the terms with even $p$ (except $p=0$ ) are removed from (10). As proposed in [10], by setting $a_{m, l}= \pm 1$ for $m=0 . . N-1$ (10) becomes

$$
a_{k, l}^{(c)}=a_{k, l} \pm j \sum_{p>0, p \text { odd }}\left(a_{k+p, l}-a_{k-p, l}\right) A_{g}\left(0, p \nu_{0}\right)
$$

where + is retained for even $l$ and - for odd $l$. To maximise the power of $a_{k, l}^{(c)}$, it is straightforward to figure out that $a_{k+p, l}$ and $a_{k-p, l}$ should have different signs, i.e., $a_{k+p, l}=-a_{k-p, l}$, for $p>0, p$ odd and for $l=0 . . N-1$. Following this rule, one example of the IAM-R preamble is shown in Fig. 1(b).

\section{B. IAM-I}

As shown in (9), the larger the power of $a_{k, l}^{(c)}$ the better the channel estimation performs. Motivated by IAM-R, a new method named IAM-I was proposed in [11] by allowing elements in the preamble sequence to be imaginary. For example if we set $a_{k, l}=j$ and $a_{k-1, l}=-a_{k+1, l}=1$ where $l$ is odd, omitting small values $A_{g}\left(0, p \nu_{0}\right), p>1$, the demodulation output in (12) becomes

$$
a_{k, l}^{(c)} \approx j\left(1+2 A_{g}\left(0, \nu_{0}\right)\right)
$$

A triplet $[1, j,-1]$ for odd $l$ was proposed to formulated the preamble sequence, as shown in Fig. 1(c), where $a_{0}, a_{1}, \ldots a_{N / 3} \in\{1,-1\}$ are randomly selected. As a result, the corresponding demodulated symbols triplet will be

$$
[(1+\delta) \pm j \delta, j(1+2 \delta),-(1+\delta) \pm j \delta]
$$

where $\delta=A_{g}\left(0, \nu_{0}\right)>0$ and $A_{g}\left(0, p \nu_{0}\right)$ for $p>1$ are omitted. For even $l$, according to (12), we should use $[-1, j, 1]$ instead, which was not discussed or even noticed in [11].

\section{IAM-C}

Observing in (14) the fact that only the center symbol of the triplet guarantees a demodulated symbol power as expected in (13), one may think of reorganising the preamble sequence so that every transmitted preamble symbol will result in the same power. Further, one may try to find a complex-valued preamble sequence so that the power of all the corresponding demodulated symbols to be maximised.

Recall the expression for $a_{k, l}^{(c)}$ in (10), with complex-valued $a_{k+p, l}$, the optimal sequence will require that all the elements in the summation should be constructively added, that is

$$
\operatorname{angle}\left(a_{k+p, l}(-1)^{p l} j^{p} A_{g}\left(0, p \nu_{0}\right)\right)=\Omega \text {, for all } k, p
$$

where $\Omega$ is an arbitrary constant within $[0,2 \pi)$. Without loss of generality, we will assume $\Omega=0$ in the following and the resulting preamble sequence will serve as a basis for a family of preamble sequences which differ each other only by a constant multiplier $e^{j \Omega}$.

Given the power constraint $\left|a_{m, n}\right| \leq 1$ and real-valued nature of $A_{g}\left(0, p \nu_{0}\right)$, we can conclude from (10) that the optimal solution that satisfies (15) for $\Omega=0$ is as follow

$$
a_{k+p, l}=(-1)^{p l} j^{-p} \cdot \operatorname{sign}\left(A_{g}\left(0, p \nu_{0}\right)\right) \text {, for all } k, p
$$

where the function $\operatorname{sign}(x)$ equals to 1 for $x \geq 0$ and -1 otherwise. Assume $A_{g}\left(0, p \nu_{0}\right) \geq 0$ holds for all $p$ (all the 


\begin{tabular}{|rrr|l|}
\hline 0 & 1 & 0 & \\
0 & $\mathrm{j}$ & 0 & \\
$\mathrm{1}$ & -1 & $\mathrm{1}$ & \\
$\mathrm{1}$ & -1 & 1 & \\
$\mathrm{1}$ & $-\mathrm{j}$ & 1 & Data \\
1 & 1 & 1 & \\
1 & 1 & 1 & \\
0 & 1 & 0 & \\
\hline
\end{tabular}

Figure 2. An example of IAM-C for odd $l$ in OFDM/OQAM.

significant terms are positive indeed), then it is easy to figure out that $a_{k, l}=j^{-k}, k=0, \ldots, N-1$ is the optimal preamble basis for even $l$ and $a_{k, l}=j^{k}$ for odd $l$, with the resulting demodulated symbol $a_{k, l}^{(c)}=\sum_{p} A_{g}\left(0, p \nu_{0}\right)$. This new method was named as IAM-C (complex) and one example of this preamble structure for odd $l$ is shown in Fig. 2.

\section{Simulation Results}

\section{A. simulation parameters}

All the simulation results in this section are carried out on the Matlab/Octave Simulation Workbench for Software Defined Radio [15], with the following main parameters of the system given below:

- Frequency separation: $F=\nu_{0}=15 \mathrm{kHz}$

- OQAM symbol duration: $\tau_{0}=33.3333 \mu \mathrm{s}$

- CP-OFDM symbol duration: $T_{o}=2 \tau_{0}+T_{c p}$

- Sampling interval: $T_{s}=0.5213 \mu \mathrm{s}$

- FFT/IFFT size: $N=128$

- CP used in OFDM: 4 to 32 samples (varies with channel)

- Frame length: $1 \mathrm{CE}$ symbol + 10 OFDM data symbols

- Channel type: ideal, time and frequency dispersive

- Number of multipaths: $<10$

- QPSK, 16QAM modulation without channel coding

- Pulse shaping filter banks with 1-tap or 5-tap per carrier

Unlike in [10], [11] where $N=2048$ FFT/IFFT was used with a convolutional channel coding at rate $1 / 2$, we simply use a relatively small FFT/IFFT size and no channel coding. The reason for this is in two-fold. On one hand we are more interested in the relative performance gain among different channel estimation methods rather than exact performance in a specific system configuration. On the other hand, large FFT size and complicated channel coding take too much time for a MATLAB based simulation on a normal PC.

Three kinds of pulse shapes are used in this simulation: the half-cosine function (1-tap), the extended Gaussian function (EGF) [16] (5-tap), and the TFL1 function [12] (1-tap). The TFL1 function was also used in [10], [11]. Note that both the EGF function and the TFL1 function are obtained from the Gaussian function $g_{\alpha}(t)=(2 \alpha)^{1 / 4} e^{-\pi \alpha t^{2}}, \alpha>0$ and therefore their localisation property will change with the variation of $\alpha$ [16].

An extended Monte-Carlo based wide sense stationary uncorrelated scattering (WSSUS) channel model [17] for doubly dispersive channels is used. With assumption of an exponential delay power profile and a classic Doppler power spectrum, the
Table I

AVERAGE POWER OF THE DEMODULATED PREAMBLE SYMBOLS FOR DIFFERENT PREAMBLE SEQUENCES

\begin{tabular}{|c|c|c|c|}
\hline$\left|a_{k, l}^{(c)}\right|^{2}$ & IAM-R & IAM-I & IAM-C \\
\hline \hline 5-tap EGF $(\alpha=1)$ & 1.2475 & 1.7978 & 2.2229 \\
\hline 5-tap EGF $(\alpha=2)$ & 1.7214 & 2.6827 & 3.4149 \\
\hline 5-tap EGF $(\alpha=7)$ & 1.9973 & 3.5207 & 3.9947 \\
\hline 1-tap TFL1 $(\alpha=1)$ & 2 & 3.1042 & 4 \\
\hline 1-tap TFL1 $(\alpha=2)$ & 2 & 3.2601 & 4 \\
\hline 1-tap TFL1 $(\alpha=7)$ & 2 & 3.5826 & 4 \\
\hline 1-tap halfcosine & 2 & 2.9844 & 4 \\
\hline
\end{tabular}

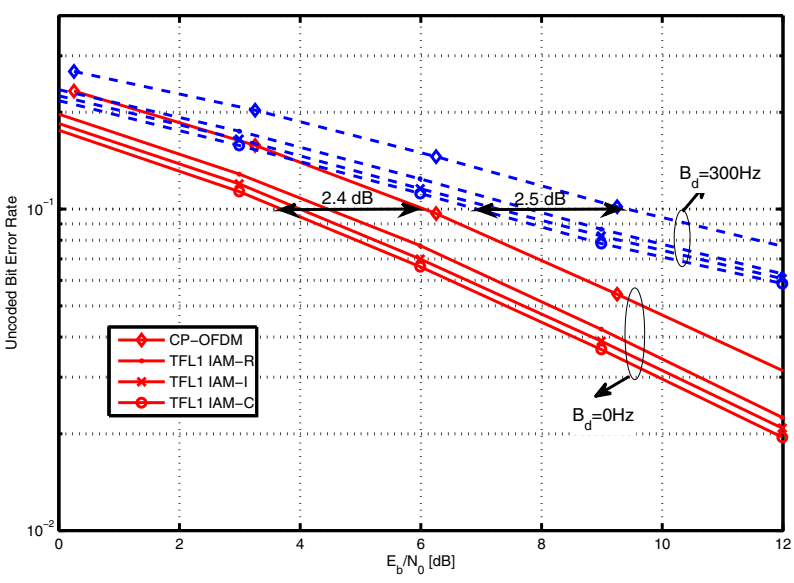

Figure 3. Uncoded BER vs. $E_{b} / N_{0}$ for CP-OFDM ( $\left.T_{c p}=T_{d}=4.167 \mu \mathrm{s}\right)$ and OFDM/OQAM with a QPSK modulation and different Doppler spread.

channel scattering function can be written as

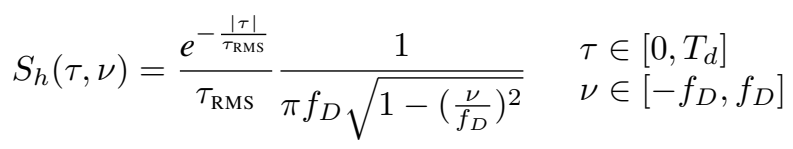

where $\tau_{\mathrm{RMS}}$ is the RMS delay spread, $f_{D}$ is the maximum Doppler frequency shift. The delay spread $T_{d}=4 \tau_{\mathrm{RMS}}$ and the Doppler spread $B_{d}=2 f_{D}$ are used in the following to describe the amount of channel dispersion.

\section{B. Power of the demodulated symbols}

The average power of the demodulated preamble symbols via an ideal channel for different IAM preamble are shown in Table I. It is clear that the IAM-C preamble always produces the highest output power and hence the best channel estimation performance. The possible influence of the Gaussian parameter $\alpha$ of different IAM methods, is interesting but outside the scope of this paper. Therefore in the following, we only use $\alpha=1$ for the EGF pulse and the TFL1 pulse in OFDM/OQAM, the same $\alpha$ value as in [10], [11].

\section{Uncoded BER over doubly dispersive channels}

Uncoded BER simulation versus $E_{b} / N_{0}$, with $E_{b}$ the useful bit energy and $N_{0}$ the mono-lateral noise density, has been carried out for different preamble sequences under dispersive channels, as shown in Fig. 3, where two dispersive channels with delay spread $T_{d}=4.167 \mu \mathrm{s}$ and different Doppler spread $(0 \mathrm{~Hz}$ and $300 \mathrm{~Hz})$ are used. With a carrier frequency 


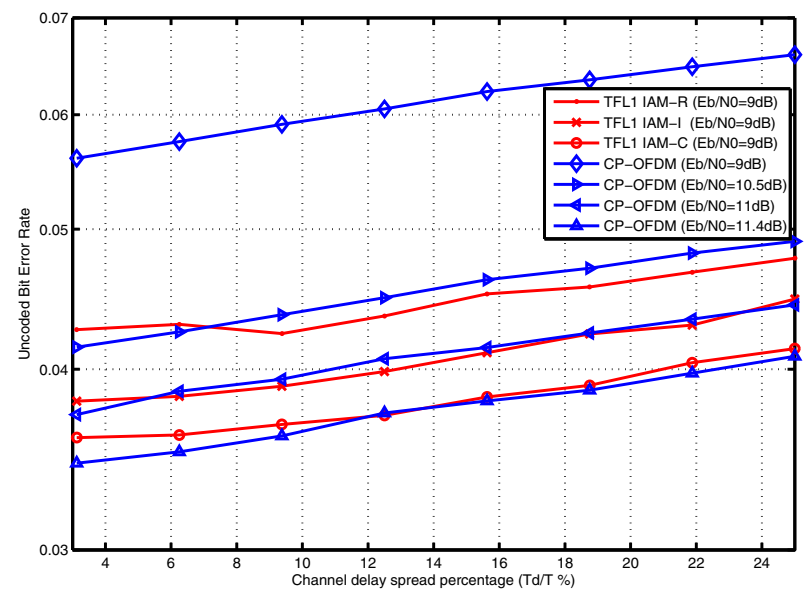

Figure 4. Uncoded BER vs. the percentage channel delay spread $T_{d} / T[\%]$ for CP-OFDM $\left(T_{c p}=T_{d}\right)$ and OFDM/OQAM with a QPSK modulation through purely time dispersive channels $\left(B_{d}=0 \mathrm{~Hz}\right)$.

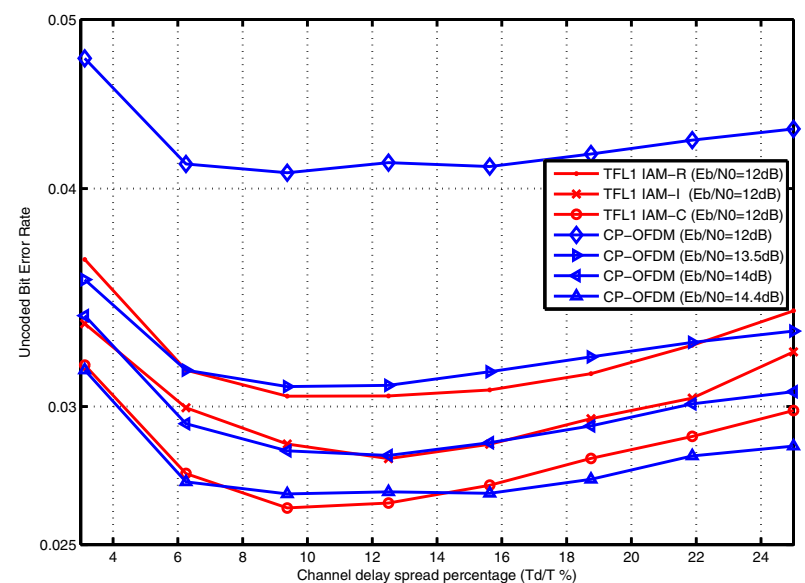

Figure 5. Uncoded BER vs. the percentage channel delay spread $T_{d} / T[\%]$ for CP-OFDM $\left(T_{c p}=T_{d}\right)$ and OFDM/OQAM with a QPSK modulation through time dispersive channels with Doppler spread $B_{d}=100 \mathrm{~Hz}$.

$f_{c}=2 \mathrm{GHz}$ and a Doppler spread $B_{d}=300 \mathrm{~Hz}$, this is equivalent to a moving speed of $81 \mathrm{~km} / \mathrm{h}$. In both cases, the length of $\mathrm{CP}$ is chosen equal to the length of the channel delay spread, i.e., $T_{c p}=T_{d}$. The IAM-C method (marker o) always outperforms CP-OFDM (marker $\diamond$ ) by at least $2.4 \mathrm{~dB}$. The gain increases slightly when Doppler shift is added. Besides, IAM-C always outperforms IAM-I (marker $\times$ ) and IAM-R (marker $\cdot$ ) by $0.4 \mathrm{~dB}$ and $0.9 \mathrm{~dB}$, respectively. A similar figure as Fig. 3 generated by increasing the channel delay spread to $T_{d}=12.5 \mu$ s gives the same conclusion, and is therefore omitted due to space limitations.

\section{Robustness against channel delay spread}

In this part we check the robustness of the IAM preambles against channel delay spread. The channel delay spread percentage is defined by $T_{d} / T$, where $T_{d}$ is the delay spread of the channel and $T=2 \tau_{0}$ is the OFDM symbol duration without adding $\mathrm{CP}$.

Fig. 4 and Fig. 5 show the uncoded BER performance with

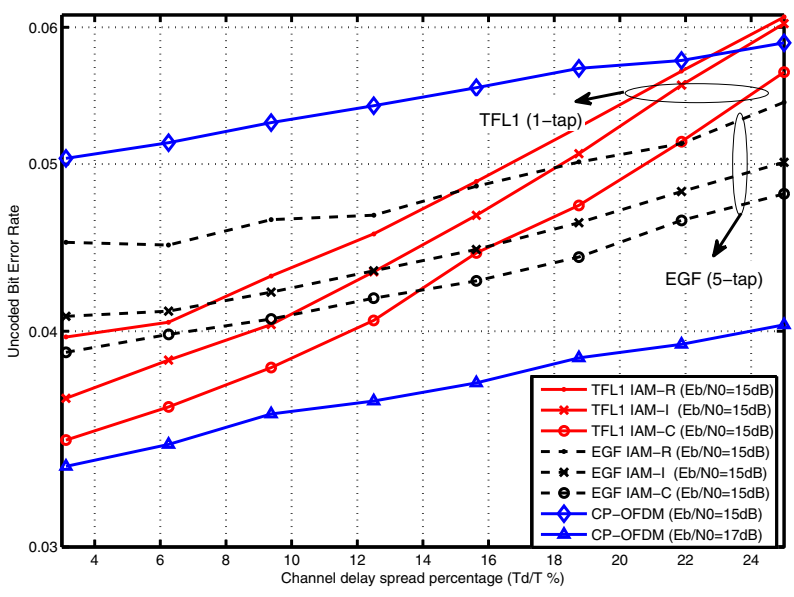

Figure 6. Uncoded BER vs. the percentage channel delay spread $T_{d} / T[\%]$ for CP-OFDM $\left(T_{c p}=T_{d}\right)$ and OFDM/OQAM with a 16-QAM modulation through purely time dispersive channels $\left(B_{d}=0 \mathrm{~Hz}\right)$.

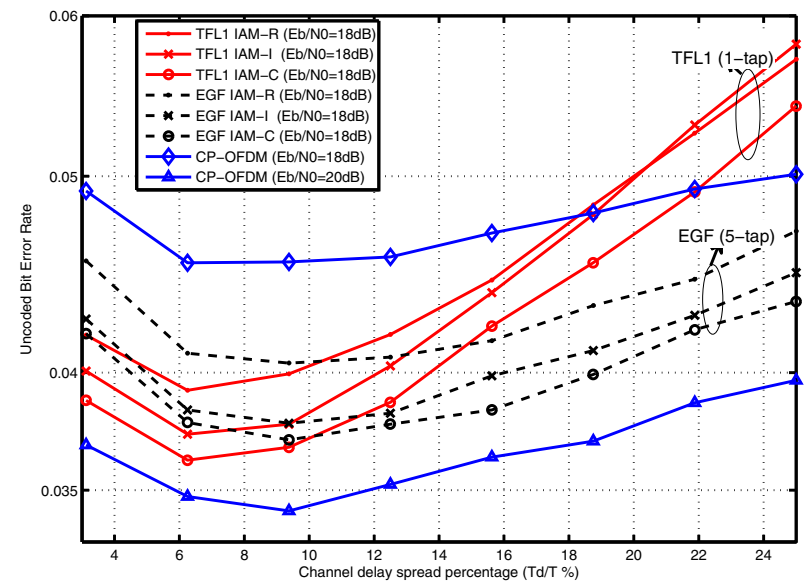

Figure 7. Uncoded BER vs. the percentage channel delay spread $T_{d} / T[\%]$ for CP-OFDM ( $\left.T_{c p}=T_{d}\right)$ and OFDM/OQAM with a 16-QAM modulation through time dispersive channels with Doppler spread $B_{d}=100 \mathrm{~Hz}$.

IAM-R, IAM-I and IAM-C versus the percentage of channel delay spread, at a given $E_{b} / N_{0}$ with a QPSK modulation. CPOFDM with different length of CP at some constant $E_{b} / N_{0}$ are also plotted as a benchmark. When the channel is purely time dispersive, as shown in Fig. 4, IAM-C can outperform CP-OFDM by $2.4 \mathrm{~dB}$ and IAM-I by $0.4 \mathrm{~dB}$ up to $25 \%$ delay spread. The gain decreases a little when the delay spread percentage is relatively small $(<6 \%)$, in which case the benefit of pulse shaping cannot be fully exploited. When there is Doppler spread present, as shown in Fig. 5, the same gains hold up to $16 \%$ delay spread. The curves for 5-tap EGF lie in the space between TFL1 and CP-OFDM, have somewhat similar shape as CP-OFDM and are therefore omitted to make the figures readable.

When a 16-QAM modulation is used, as shown in Fig. 6 and Fig. 7, a notable gain degradation happens for all the IAM based methods in OFDM/OQAM. The benefit of IAMC compared to IAM-R and IAM-I is somehow kept. Besides, when TFL1 is used the gain over CP-OFDM diminishes 


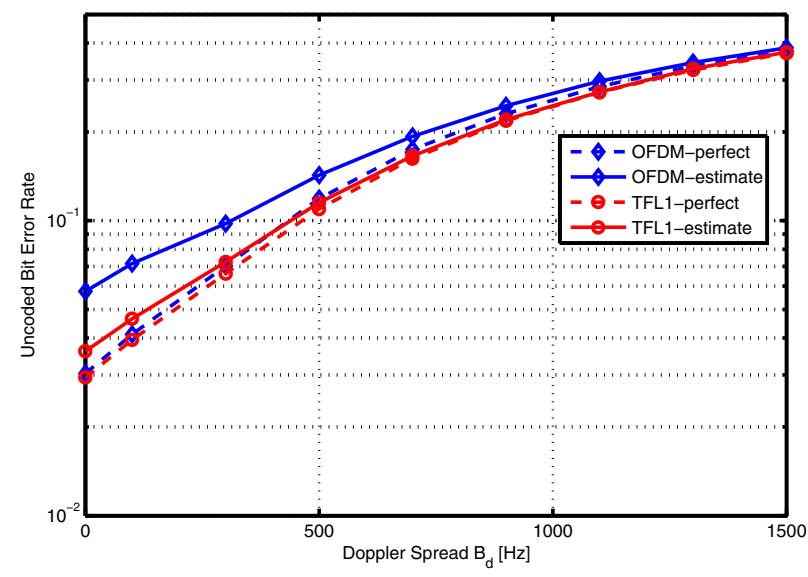

Figure 8. Uncoded BER vs. Doppler spread $B_{d}[\mathrm{~Hz}]$ for CP-OFDM $\left(T_{c p}=T_{d}=4.167 \mu \mathrm{s}\right)$ and OFDM/OQAM (TFL1 with IAM-C) with a QPSK modulation, $E_{b} / N_{0}=9 \mathrm{~dB}$.

quickly as the time spread percentage increases. However, it changes relatively slow for 5-taps EGF, which always outperforms CP-OFDM in time dispersive channels (up to $25 \%$ ) with or without Doppler spread. The results here also indicate the necessity of pulse shape adaptation depending on the channel conditions. For example, it is better to use TFL1 pulse shapes in low time dispersive channels (up to $14 \%$ for $B_{d}=0 \mathrm{~Hz}$ and up to $10 \%$ for $B_{d}=100 \mathrm{~Hz}$ ) and 5-tap EGF pulse shapes otherwise.

The "U" shape curves with respect to the increase of delay spread in Fig. 5 and Fig. 7 can be interpreted as follows: The number of resolvable fading paths is very limited for small delay spread, which makes the system ICI dominated. The increase of delay spread in this scenario will provide extra paths and therefore increase the gain of diversity. When delay spread becomes larger and larger, the system becomes ISI dominated, which gradually degrades the system performance, especially for OFDM/OQAM.

\section{E. Robustness against channel Doppler spread}

The robustness of the IAM preambles against channel Doppler spread over doubly dispersive channels is reported in Fig. 8. Compared with the case of "pseudo perfect" channel estimation [9] (dashed lines), TFL1 with IAM-C preamble experiences very limited degradation. CP-OFDM on the other hand suffers a lot from the Doppler spread. However, the performance of CP-OFDM and OFDM/OQAM degrades very fast as the increase of Doppler spread, which makes it necessary to insert CE preambles into the data frame several times when Doppler spread is large.

\section{CONCLUSiON}

In this paper we have presented a general theoretical framework for preamble-based IAM channel estimation methods for OFDM/OQAM systems. We also use this framework to revisit the two heuristic IAM structures and have identified the optimal IAM preamble sequence. It has been shown that, when QPSK is used, the proposed optimal structure IAM$\mathrm{C}$ can achieve about $2.4 \mathrm{~dB}$ gain over CP-OFDM in doubly dispersive channels up to $25 \%$ delay spread with no Doppler spread and up to $16 \%$ delay spread with Doppler spread $B_{d}=100 \mathrm{~Hz}$. Compared to IAM-R and IAM-I, a moderate gain is always maintained of $0.9 \mathrm{~dB}$ and $0.4 \mathrm{~dB}$, respectively. When 16-QAM is used, the corresponding gain becomes smaller, and these pulse shapes with better TFL properties are preferred when the channel is largely time dispersive.

All the IAM methods discussed in this paper require the calculation of the "pseudo pilot", which can be done offline if the pulse shape and preamble are predefined. Since the IAM preamble structure takes 3 OFDM/OQAM symbols, i.e., one and a half OFDM symbols, it is not very attractive for high mobility communications, where the preamble has to be inserted frequently. On the other hand, preliminary results for more advanced CE methods have shown the usefulness of this framework in preamble analysis and design. Therefore we will focus on time and energy efficient preamble design methods in future work based on the proposed framework.

\section{REFERENCES}

[1] R. W. Chang, "Synthesis of Band-Limited Orthogonal Signals for Multicarrier Data Transmission," Bell. Syst. Tech. J., vol. 45, pp. 1775-1796, Dec. 1966.

[2] B. Hirosaki, "An Orthogonally Multiplexed QAM System Using the Discrete Fourier Transform", IEEE Transactions on Communications, vol. 29, no. 7, pp. 982-989, Jul. 1981.

[3] B. le Floch, M. Alard and C. Berrou, "Coded Orthogonal Frequency Division Multiplex," Proceedings of the IEEE, pp. 982-996, Jun. 1995.

[4] P. Jung, G. Wunder and C. S. Wang, "OQAM/IOTA Downlink Air Interface for UMTS HSDPA Evolution," 9th International OFDM-Workshop, Hamburg, pp. 153-157, 2004.

[5] M. Bellec and P. Pirat, "OQAM performances and complexity," IEEE P802.22 Wireless Regional Area Network, Jan. 2006.

[6] TIA Committee TR-8.5, "Wideband Air Interface Isotropic Orthogonal Transform Algorithm (IOTA) -Public Safety Wideband Data Standards Project - Digital Radio Technical Standards," 2003

[7] J.P. Javaudin, D. Lacroix and A. Rouxel, "Pilot-aided channel estimation for OFDM/OQAM," VTC'03 Spring, pp. 1581-1585, Apr. 2003.

[8] S.W. Kang and K.H. Chang, "A novel channel estimation scheme for OFDM/OQAM-IOTA system," ETRI Journal, vol. 29, pp. 430-436, Aug. 2007.

[9] D. Lacroix and J.P. Javaudin, "A new channel estimation method for OFDM/OQAM," 7th International OFDM-Workshop, Sep. 2002.

[10] C. Lélé, J.P. Javaudin, R. Legouable, A. Skrzypczak and P. Siohan, "Channel estimation methods for preamble-based OFDM/OQAM modulations," European Wireless Conference, Paris, France, Apr. 2007.

[11] C. Lélé, P. Siohan and R. Legouable, " $2 \mathrm{~dB}$ better than CP-OFDM with OFDM/OQAM for preamble-based channel estimation," IEEE International Communication Conference, Beijing, China, Apr. 2008.

[12] P. Siohan, C. Siclet and N. Lacaille, "Analysis and design of OFDM/OQAM. systems based on filterbank theory", IEEE Transactions on Signal Processing, vol. 50, no. 5, pp. 1170-1183, May 2002.

[13] H. Bölcskei, "Orthogonal Frequency Division Multiplexing Based on Offset-QAM", Advances in Gabor Analysis, Birkhäuser, 2003.

[14] L. Vangelista and N. Laurenti, "Efficient Implementations and Alternative Architectures for OFDM-OQAM Systems", IEEE Transactions on Communications, vol. 49, no. 4, pp. 664-675, Apr. 2001.

[15] S. Signell and J. Huang, "A Simulation Environment for Multi-Antenna Software Defined Radio," in Proc. of ICICS07, Singapore, Dec. 2007.

[16] P. Siohan and C. Roche, "Cosine-Modulated Filterbanks Based on Extended Gaussian Function," IEEE Transactions on Signal Processing, vol. 48, no. 11, pp. 3052-3061, Nov. 2000.

[17] P. Hoeher, "A Statistical Discrete-Time Model for the WSSUS Multipath Channel," IEEE Transactions on Vechicular Technology, vol. 41, no. 4, pp. 461-468, Nov. 1992. 